\section{REVISTA BRASILEIRA DE QUALIDADE DE VIDA}

\title{
Qualidade de vida de estudantes de enfermagem de uma faculdade privada
}

\author{
Quality of life of nursing students from a private university
}

\author{
Everton Fernando Alves \\ Universidade Estadual do Norte do Paraná - UENP - Jacarezinho - Brasil \\ Clínica de Cirurgia Plástica Dr. Fábio Paixão - CCPDFP - Maringá - Brasil \\ evertonando@hotmail.com
}

\begin{abstract}
Resumo
O presente estudo teve como objetivo avaliar a qualidade de vida dos estudantes no curso de Enfermagem de uma faculdade privada. Realizou-se um estudo descritivo, com abordagem quantitativa, utilizando a Escala de Qualidade de Vida de Flanagan e um instrumento de coleta de dados complementares, contendo questões estruturadas. Resultou numa amostra de 192 (cento e noventa e dois) estudantes. O diagnóstico evidenciou que a maioria dos estudantes está 'Satisfeitos' e 'Pouco satisfeitos' com a sua qualidade de vida, entretanto identificou a presença de vários problemas que necessitam de atenção dos educadores. Concluiu-se que se faz necessária a formação de um núcleo de estudos interdisciplinar, de pesquisa e extensão sobre Qualidade de Vida (QV), proporcionando apoio aos estudantes no que diz respeito aos aspectos imprescindíveis apresentados no estudo, para melhoria de suas QV. Isto pode preparar e adaptar o futuro profissional à nova etapa de vida que está apenas se iniciando.
\end{abstract}

Palavras-chave: qualidade de vida, estudantes, enfermagem.

\begin{abstract}
This study was designed with the objective of evaluating the quality of life of students in the nursing course of a private college. We conducted a descriptive study with a quantitative approach, using the Scale of Quality of Life of Flanagan and an instrument to collect additional data, with questions structured. Resulted in a sample of 192 (one hundred ninety-two) students. The diagnosis showed that most students are "satisfied" or " median level of satisfaction" with their quality of life, however identified the presence of several problems that need attention from educators. It was concluded that it is necessary to form a core of interdisciplinary studies, research and extension on Quality of life (QOL), providing support to students with regard to vital aspects presented in the study to improve their QOL. This can prepare and adapt to future professional new stage of life that is just the beginning.
\end{abstract}

Keywords: quality of life, students, nursing.

\section{Introdução}

A entrada do estudante na academia de enfermagem, muitas vezes, exige adaptações a uma nova realidade tanto a nível pessoal, social, quanto acadêmico. A transição do aluno, do ensino secundário ao ensino superior, faz com que o mesmo se depare com novas exigências, em relação às 
tarefas que a faculdade exige do estudante, como a adoção de novos comportamentos (CORREIA, 2003).

Essa fase universitária se torna de extrema importância ao se considerar que o jovem estará tendo contato com múltiplos fatores, que farão com que este se desenvolva pessoal e profissionalmente. Entretanto, quando não ocorre a adaptação e a sensação de realização - enquanto futuro profissional -, ocorre o insucesso acadêmico, traduzido por problemas com os estudos, sendo o meio utilizado pelo estudante que revela o seu desconforto emocional (CORREIA, 2003).

Não obstante, existem hipóteses que comentam as situações desfavoráveis que afetam a QV dos estudantes de Enfermagem, como por exemplo, sofrimento no viver genérico relacionado a esta fase de vida, que faz surgir problemas financeiros, familiares, de saúde, e também no cotidiano específico do curso, que carrega sentimentos íntimos de sofrimento decorrente da dor e da morte de pessoas que necessitam de seu cuidado. Nessa fase é comum os estudantes ao realizarem atividades práticas, não se sentirem preparados, gerando neles sentimentos como: estresse, medo, ansiedade, inexperiência em lidar com situações críticas e desgastes físicos (SAUPE, 2002).

Percebe-se também que o perfil dos estudantes de enfermagem é o de ser "trabalhadorestudante", enfrentando, na grande maioria, dupla ou tripla jornada de trabalho. Em consequiência das más condições de vida, apresentadas freqüentemente por eles, chegam atrasados ou dormem durante as aulas, podendo interferir em sua qualidade de vida e consequentemente no processo de ensino-aprendizagem (RIBEIRO; PEDRÃO, 2005). Tais fatores nem sempre são acompanhados pelo sistema de ensino, uma vez que seria essencial um apoio a estes futuros cuidadores, já que há tanto discurso humanístico nas salas de aulas (SAUPE, 2002).

Acredita-se que toda essa problemática tem relação direta com a qualidade de vida e, diante disso, torna-se imprescindível criar mecanismos de suporte que auxiliem os estudantes ao enfrentamento das inúmeras situações conflitantes que vivenciam durante o processo de formação profissional, sem que, para isso, precisem desumanizar-se (SAUPE et al., 2003).

O Grupo de Qualidade de Vida da OMS conceitua qualidade de vida como sendo a percepção do indivíduo sobre a sua posição na vida, como também no contexto da cultura e do sistema de valores nos quais ele vive e, ainda, em relação a seus objetivos, expectativas, padrões e preocupações (THE WHOQOL GROUP, 1995).

Já a Qualidade de Vida dos Estudantes (QVE) tem sido entendida como a "percepção de satisfação e felicidade, por parte do estudante em relação a múltiplos domínios de vida à luz de fatores psicossociais e contextuais relevantes e estruturas de significados pessoais" (OLIVEIRA; CIAMPONE, 2008, p. 58).

Partindo dessa premissa, percebe-se a relevância que estes aspectos têm para os futuros profissionais da enfermagem e para que o exercício da enfermagem atenda às necessidades do ser humano a ser cuidado por eles, é fundamental que os estudantes tenham conhecimento a cerca da qualidade de vida e a importância desta em suas vidas, pois antes de cuidar de outros, é imprescindível o cuidar de si (OLIVEIRA; CIAMPONE, 2008).

Diante disso, buscou-se nesse estudo avaliar a qualidade de vida de estudantes do curso de Enfermagem de uma instituição privada segundo a Escala de Flanagan.

\section{Métodos}

Trata-se de um estudo com delineamento descritivo, com abordagem quantitativa, tendo como população de estudo os estudantes matriculados nos quatro anos do curso de Enfermagem da faculdade Uningá no município de Maringá - PR, no ano de 2006. Os dados foram coletados por meio de um questionário auto-aplicável, chamado Escala de Qualidade de Vida de Flanagan que conceptualiza qualidade de vida a partir de cinco dimensões mensuradas através de quinze itens, dispostos em: 1) Bem-estar físico e material (itens 1 e 2); 2) Relacionamento com outras pessoas (itens 3, 4, 5 e 6); 3) Atividades sociais, comunitárias e cívicas (itens 7 e 8); 4) Desenvolvimento pessoal e realização (itens 9, 10, 11 e 12); e 5) Recreação (itens 13, 14 e 15) (LENTZ et al., 2000). 
Em cada item o respondente tem sete opções de resposta, que vai de "muito insatisfeito" (escore 1) até "muito satisfeito" (escore 7). A pontuação máxima alcançada é de 105 pontos e a mínima de 15 pontos, que refletem a mais alta e a mais baixa qualidade de vida respectivamente. Para se obter o valor final, foram feitas as somatórias das notas atribuídas a cada um dos itens e divididas por 15. Assim, cada nota referiu-se ao grau de satisfação do estudante, com a seguinte correspondência: muito insatisfeito $=1$, insatisfeito $=2$, pouco insatisfeito $=3$, indiferente $=4$, pouco satisfeito $=5$, satisfeito $=6$ e muito satisfeito $=7$.

Foi realizada a coleta de dados através de questões estruturadas, relacionadas à caracterização dos estudantes em estudo (idade, sexo, estado civil e profissão) e questões abertas referentes à percepção sobre QV com duas questões norteadoras: 1) "O que é "Qualidade de Vida" para você?"; e 2) "O curso de enfermagem interfere de alguma forma, positiva ou negativa, na sua qualidade de vida?".

Os procedimentos para coletas de dados incluíram: aprovação do projeto pelo diretor acadêmico da instituição; contatos com docentes solicitando autorização e agendamento para aplicar o instrumento em sala de aula, apresentação da proposta aos alunos presente no momento da coleta, com rápida explanação sobre os objetivos do estudo, o método de coleta de dados, importância da colaboração e questões éticas envolvidas (adesão voluntária, sigilo, anonimato, contribuição do estudo...). Neste momento foi apresentada e solicitada a assinatura do termo de consentimento livre e esclarecido aos participantes.

A coleta de dados foi realizada no mês de Maio de 2006, sendo efetuada pelos próprios autores do trabalho. $\mathrm{O}$ desenvolvimento do estudo ocorreu em conformidade com o preconizado pela Resolução 196/96 do CNS.

As questões abertas foram analisadas e codificadas por agrupamento de similaridade entre as respostas e a organização dos dados foi efetivada através de sua transferência para uma planilha do Excel. A análise dos dados realizou-se através da construção de tabelas e gráficos estatísticos, utilizando percentuais simples.

\section{Resultados e discussão}

Dos 243 estudantes matriculados no curso de graduação em Enfermagem da instituição em estudo no ano de 2006, 194 discentes participaram do estudo, visto que 45 não estavam presentes na sala de aula nos períodos destinados à coleta de dados e 04 se recusaram a participar do estudo e dois foram excluídos por não responderem todo o instrumento de coleta de dados resultando numa amostra de 192 estudantes (79\%).

Tabela 1 - Distribuição do total dos estudantes de enfermagem segundo variáveis sócic
\begin{tabular}{lcc}
\multicolumn{4}{c}{ UNINGÁ, Maringá-PR, 2006. } \\
\hline Variáveis & N & \% \\
\hline Idade & 45 & 23,0 \\
$<20$ anos & 108 & 56,3 \\
$20-29$ & 31 & 16,3 \\
$30-39$ & 8 & 4,4 \\
40 e mais & & \\
Estado civil * & 134 & 70,0 \\
Solteiros & 48 & 25,0 \\
Casados & 5 & 3,0 \\
Divorciados & & \\
Sexo & 54 & 80,0 \\
Feminino & 154 & 20,0 \\
Masculino & 38 & \\
Atividade & &
\end{tabular}




\begin{tabular}{lcc} 
Só estuda & 102 & 53,0 \\
Trabalha e estuda & 90 & 47,0 \\
Profissão** & 77 & 40,0 \\
Aux. Enferm. & 22 & 11,0 \\
Téc. Enferm. & 14 & 7,0 \\
Vendedores & 79 & 41,0 \\
Outra & Fonte: Pesquisa de campo (2006) \\
\hline \multicolumn{3}{c}{ * Três \% dos respondentes não informaram } \\
** Um \% dos respondentes não informou
\end{tabular}

Quanto à distribuição dos estudantes segundo a idade, nota-se que a idade média dos acadêmicos foi 24,6 anos, com idade mínima de 17 e máxima de 53 anos. Na Tabela 1, observa-se que a maioria dos acadêmicos (56,3\%) apresentava de 20 a 29 anos de idade. Entretanto ao agrupar os estudantes com mais de 30 anos, encontrou-se um total de 39 (20,7\%), considerados uma amostra relevante no estudo.

Segundo o estado civil, observa-se que $70 \%$ encontram-se solteiros enquanto $25 \%$ são casados e 3\% são os divorciados. Dos que trabalham 38,5\% são solteiros e $60,4 \%$ casados. A maior parte da amostra (80\%) relaciona-se ao sexo feminino e quase metade dela (47\%), trabalha além de estudar e 53\% não exercem nenhuma atividade relacionada. Em relação ao sexo masculino, 76,9\% destes trabalham, além de estudar. Quanto à profissão, $40 \%$ dos estudantes são auxiliares de enfermagem, $11 \%$ técnicos de enfermagem, $7 \%$ são vendedores, $41 \%$ de outras profissões e $1 \%$ não responderam.

$\mathrm{Na}$ Tabela 2 são apresentados os resultados da totalidade de respondentes, segundo as dimensões componentes da Escala de Flanagan, distribuídos conforme percentuais.

Tabela 2 - Distribuição das respostas em freqüências relativas.

\begin{tabular}{|c|c|c|c|c|c|c|c|c|}
\hline \multirow{2}{*}{ Dimensões } & \multicolumn{7}{|c|}{ Escala } & \multirow{2}{*}{ Total } \\
\hline & 1 & 2 & 3 & 4 & 5 & 6 & 7 & \\
\hline Bem-estar físico e material & 1,8 & 6,8 & 10,4 & 3,1 & 18,2 & 45 & 14,7 & 100 \\
\hline Relacionamento com outras pessoas & 5,6 & 5 & 4,7 & 11 & 12,9 & 36,6 & 24,2 & 100 \\
\hline $\begin{array}{l}\text { Atividades sociais, comunitárias e } \\
\text { cívicas }\end{array}$ & 7,7 & 9,5 & 11,3 & 15,5 & 20 & 26 & 10 & 100 \\
\hline Desenvolvimento pessoal e realização & 6,4 & 9,1 & 10,8 & 6,9 & 20,7 & 32,4 & 13,7 & 100 \\
\hline Recreação & 5,4 & 7,1 & 8,7 & 5,9 & 15,6 & 31,5 & 25,8 & 100 \\
\hline $\begin{array}{l}\text { Todas as dimensões } \\
\text { (Questões de } 1 \text { a 15) }\end{array}$ & 5,3 & 7,4 & 8,7 & 8,4 & 17,3 & 34,3 & 18,6 & 100 \\
\hline
\end{tabular}

Fonte: Pesquisa de campo (2006)

Legenda: 1 = muito insatisfeito; 2 = insatisfeitos; 3 = pouco insatisfeito; 4 = indiferente; $5=$ pouco satisfeitos $; 6=$ satisfeito $7=$ muito satisfeito.

Chama a atenção na Tabela 2 que, ao agrupar o nível 6 e 7 - Satisfeito e Muito satisfeito -, encontrou-se a maior proporção de respostas positivas (61\%) na dimensão "Relacionamento com outras pessoas", seguido da dimensão "Bem-estar físico e material" com 60\%, e o menor percentual ainda nesses níveis de satisfação foi na dimensão "atividades sociais, comunitárias e cívicas" (36\%). Apesar de ser o menor percentual, esse resultado fica fortalecido por ser a maior proporção nesta dimensão. 
Estes resultados vão de encontro ao estudo realizado por Saupe (2002) que sugere que para se formar enfermeiros com consciência de cidadania e raciocínio crítico torna-se essencial ter espaço e reforço, não só através de atividades curriculares, como também extracurriculares, para que assim não se continue formando enfermeiros técnicos e nem sempre comprometidos.

Figura 1 - Média de pontuação na Escala de Flanagan segundo os escores atribuídos ao grau de satisfação dos estudantes. UNINGÁ, Maringá-PR, 2006.

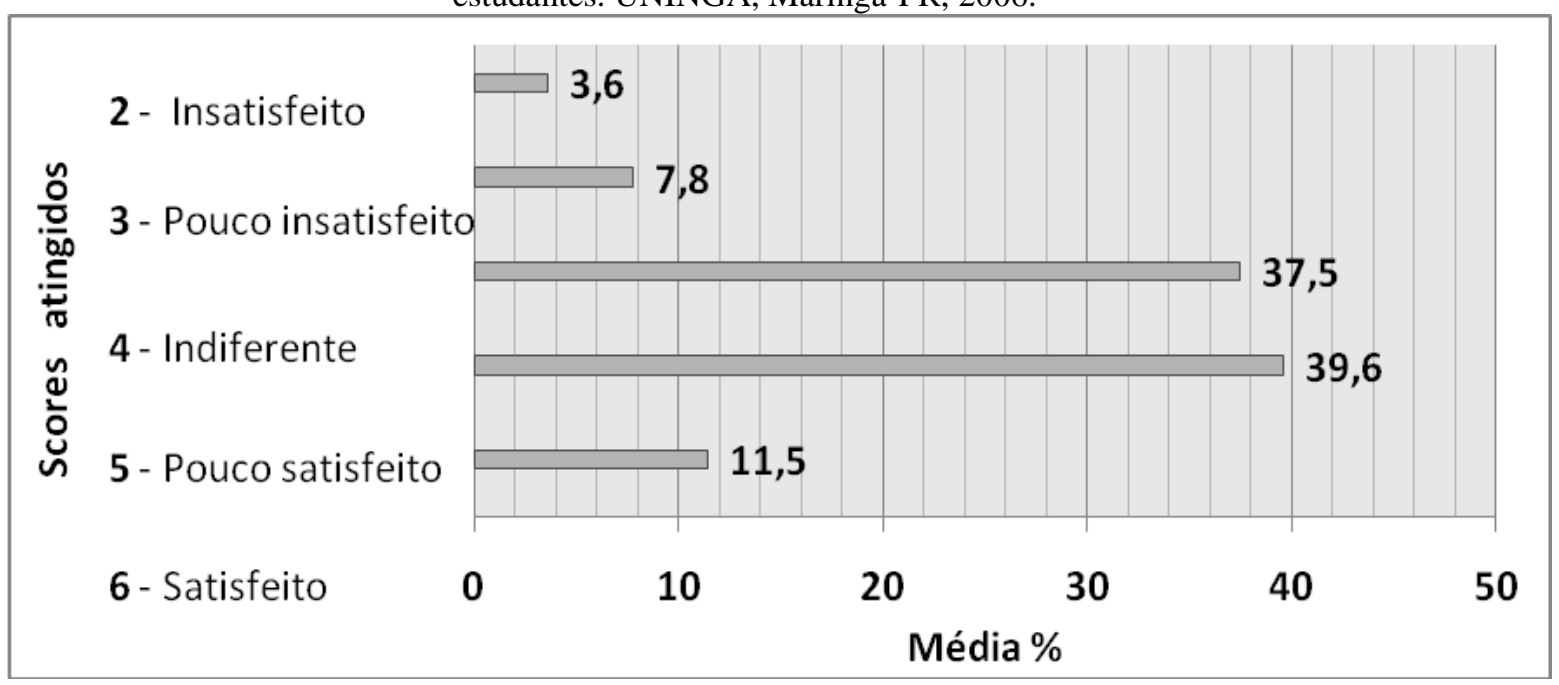

Fonte: Pesquisa de campo (2006)

Ao analisar, na Figura 1, a média geral dos scores atribuídos pelo grupo de estudantes a cada item do instrumento, observa-se que a maior proporção $(51,4 \%)$, incluindo os 'Satisfeitos' e 'Pouco satisfeitos' se encontra em um grau de satisfação que pode ser considerado como uma qualidade de vida que varia entre razoável a boa. Entretanto chama a atenção o percentual de 48,9\%, incluindo os 'Insatisfeitos', 'Pouco insatisfeitos' e 'Indiferentes', os quais podem ser considerados alto e preocupante, sugerindo a necessidade de investigação mais dirigida à individualidade dos sujeitos.

Nesta análise geral, nota-se que não houve a presença de pontuações relativas a "Muito satisfeito" e "Muito insatisfeito". Isto pode estar relacionado ao fato de que os estudantes de enfermagem, por cursarem em uma instituição privada, possuem condições de vida que os mantém em um grau de satisfação nivelado entre os dois extremos.

Figura 2 - Opinião dos estudantes de enfermagem segundo a interferência positiva do curso na qualidade de vida. UNINGÁ, Maringá-PR, 2006.

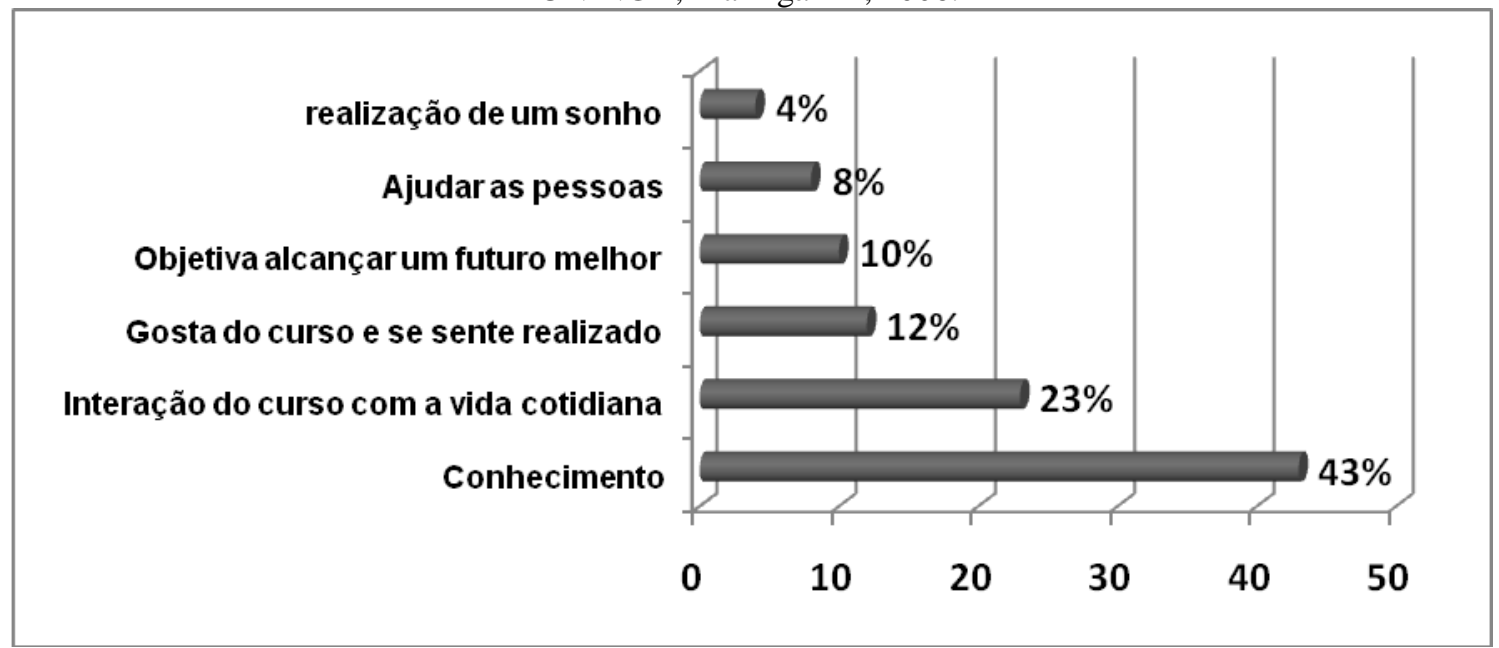

Fonte: Pesquisa de campo (2006)

Ao responderem à pergunta sobre a interferência de forma positiva do curso de enfermagem em sua vida, encontrou-se coerência com as respostas dadas às questões fechadas. De acordo com a 
Figura 2, nota-se que, $43 \%$ dos estudantes dizem que o curso de enfermagem influencia de maneira positiva em sua qualidade de vida por proporcionar aumento em seus conhecimentos, $23 \%$ relatam uma experiência positiva em relação à interação do curso com a vida cotidiana e, $12 \%$ se sentem realizado na escolha do curso.

O aprendizado que o curso de enfermagem proporciona ao estudante representa uma experiência nova, de oportunidades de reescreverem seus caminhos e suas metas, tornando-se responsável pelo seu próprio aprendizado. Os estudantes consideram essa experiência significativamente positiva, na qual eles podem desenvolver-se pessoal e profissionalmente, imbuídos da reflexão crítica e da capacidade de buscar novos caminhos. Quando estes alcançam as metas pré-estabelecidas por si próprios, evidenciam em seu viver as influências positivas, tornandose mais confiantes, aprendendo a planejar suas atividades e revestindo-se de autonomia ao direcionar seus próprios aprendizados, o que os fazem acreditar que vai levar esse aprendizado para todos os âmbitos de vida (OLIVEIRA; CIAMPONE, 2008; PETTENGILL; NUNES; BARBOSA, 2003).

Figura 3 - Opinião dos estudantes de enfermagem segundo a influência negativa do Curso na qualidade de vida. UNINGÁ, Maringá-PR, 2006.

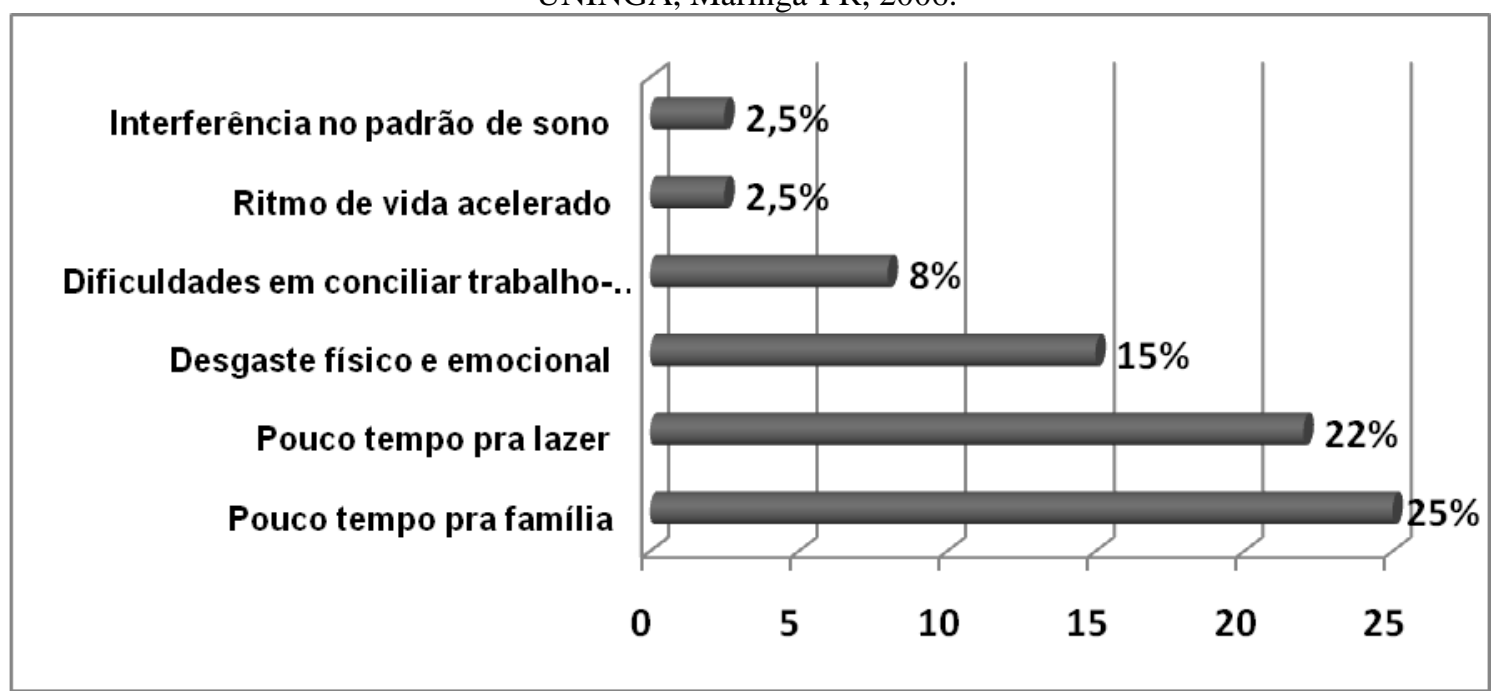

Fonte: Pesquisa de campo (2006)

Na Figura 3 observam-se a inclusão de aspectos negativos do curso na qualidade de vida dos estudantes. Estas negatividades concentraram-se em queixas relacionadas a terem pouco tempo para conviverem com a família (25\%) e pouco tempo para o lazer (22\%). Ainda cabe ressaltar que $15 \%$ das respostas referem-se à presença de ansiedade e estresse na vida dos estudantes.

Dados semelhantes são encontrados em estudos similares mostrando que a qualidade de vida de estudantes tem sido negativamente influenciada por estresse e ansiedade, decorrentes de atividades práticas para as quais não se sentem preparados e da interferência das horas exigidas para estudos teóricos (OLIVEIRA; CIAMPONE, 2008; SAUPE, 2002). Ocorrem ainda conflitos pelas necessidades afetadas por afastamento do meio familiar, sono e repouso, exercícios e atividades físicas, nutrição e conflitos nas relações interpessoais. Muitas vezes o estudante de enfermagem é obrigado a permanecer cerca de nove horas diárias de atividades de ensino, situação semelhante à jornada integral de trabalho (SANTOS et al., 2000).

Pode-se ainda inferir que toda essa problemática interfere no padrão de sono dos estudantes, podendo afetar a qualidade de vida e o seu processo de aprendizado. Estudos demonstram que os estudantes de enfermagem dormem menos de 8 horas de sono consideradas ideais por noite, apresentando um sono insuficiente e de baixa qualidade (PIZÃO; MARTINO, 2005; IGLESIAS, 2002). Percebe-se ainda que os estudantes que trabalhavam no período noturno (plantões) dormiam aproximadamente $05 \mathrm{~h} 13 \mathrm{~min}$ por noite durante a jornada, todos eles trabalhavam conforme esquema de plantões noturnos de12 horas, não sendo sempre possível dormir durante a jornada de trabalho 
ou depois da mesma, fazendo com que os estudantes que trabalhavam, dormisse menos que os que não trabalham.

Nos dias úteis, estes estudantes dormiam tarde e acordavam cedo, motivo este considerado como causa provável do déficit de sono, que ia se acumulando no decorrer da semana, causando um sono noturno de baixa qualidade e uma sensação de persistência do cansaço, ao acordar. O sono tem sido considerado tema preocupante ao analisar pesquisas envolvendo estudantes de enfermagem, que demonstram a sua maioria $(70,5 \%)$ não conseguindo manter a atenção durante as aulas devido ao cansaço e sonolência, afetando diretamente a qualidade de vida e o aprendizado.

Observa-se que o conceito de qualidade de vida, expresso por $27,6 \%$ dos estudantes de enfermagem compreende a qualidade de vida como sendo "boa alimentação, realização profissional, lazer, exercício físico, amigos, família e sono" e 22, 9\% percebem-na como "bem estar psicológico, social, físico, espiritual e ter lazer". Diante disso, pode-se perceber que apesar da subjetividade dos discursos emitidos pelos estudantes, estes demonstram sua percepção baseada em princípios sustentados pelo senso comum, sendo perceptível quando comparadas estas definições com conceitos de diversos autores apresentados na literatura, podendo constatar que a QVE depende fundamentalmente da própria avaliação que fazem de sua vida e, portanto não se pode extrair uma definição exata dos relatos dos estudantes de enfermagem.

\section{Conclusão}

A análise referente à caracterização da população possibilitou verificar que a presença de alunos com mais de 30 anos, perfazendo $20,7 \%$, demonstra que a faixa etária no curso de enfermagem da instituição em estudo é bastante diversificada e que pessoas com idade mais adulta estão procurando qualificar-se para enfrentar o mercado de trabalho cada vez mais competitivo.

O diagnóstico evidenciou através dos resultados obtidos na Escala de Flanagan, que a maioria dos estudantes está 'Satisfeitos' e 'Pouco satisfeitos' com a sua qualidade de vida. Os resultados obtidos nas questões abertas evidenciaram que os estudantes referem como positiva a "interação do curso com a vida cotidiana" e "aquisição de conhecimento" e, negativa em relação "tempo para lazer" e "tempo para com a família".

Ao se analisar os conceitos de qualidade de vida emitidos pelos estudantes de enfermagem verificaram-se similaridades entre os atributos conceituados pelos mesmos, o que pode significar que independentemente do padrão social, há concordância sobre o que consideram fundamentais para o seu bem viver.

Em relação ao lazer, sugere-se que os estudantes de enfermagem, adotando uma nova forma de pensar, possam rever o significado de lazer que tem para si, adotando uma visão holística do lazer em sua vida profissional e social, seja em momentos isolados da atividade acadêmica ou intrínseca às ações de qualquer natureza. Independente de entendimento ou manifestação espaçotemporal, o ideal seria que ele fosse tomado e valorizado como um requisito para o cuidado de si, capaz de trazer conforto, bem-estar, alívio, alegria, tranqüilidade... E, assim, oportunizar o cuidado de outros, em qualquer espaço de tempo que esse encontro aconteça.

Mas, este estudo pode, igualmente, trazer novos rumos que vão para além do conhecimento técnico-científico. Deste modo, seria muito interessante alargar esta pesquisa a outros cursos da faculdade em pesquisa. Uma vez que cada curso tem as suas especificidades que caracterizam e determinam os estudantes que nela se encontram. Seria pertinente repetir a pesquisa noutros cursos, percebendo até que ponto existe diferenças e semelhanças entre os cursos, entre os estudantes e entre os empecilhos para obter um nível elevado de qualidade de vida.

Sendo o nível da qualidade de vida dos estudantes de enfermagem um tema preocupante, um trabalho deste gênero irá contribuir para compreendê-lo melhor, bem como qual o papel que desempenha e a possível contribuição deste tipo de enfoque na promoção do aumento do nível da qualidade de vida.

Os estudantes de faculdades privadas precisam também ser conscientizados sobre a importância do "cuidar de si" através do incentivo ao exercício físico e da alimentação balanceada voltada às atividades práticas realizadas por eles, ainda em estágio, através de orientações 
individuais ou em grupo. Isto pode preparar e adaptar o futuro profissional à nova etapa de vida que está apenas se iniciando.

Faz-se necessária a formação de um núcleo de estudos interdisciplinar, de pesquisa e extensão sobre qualidade de vida, proporcionando apoio aos estudantes no que diz respeito a lidarem com a alimentação e a prática de atividade física, desmotivando o uso de álcool e fumo e alertando sobre os malefícios e a influência da propaganda.

\section{Referências}

CORREIA, T. S. O insucesso escolar no ensino superior: estudo de caso. 2003. 154 f. Tese (Doutorado em Sociologia) - Instituto Superior de Ciências do Trabalho e da Empresa, Lisboa.

IGLESIAS, R. B. Qualidade de vida de alunos-trabalhadores que cursam a graduação em enfermagem. 2002. 97 f. Dissertação (Mestrado em Enfermagem) - Escola de Enfermagem, Universidade de São Paulo, São Paulo.

LENTZ, R. A. et al. O profissional de enfermagem e a qualidade de vida: uma abordagem fundamentada nas dimensões propostas por Flanagan. Revista Latino-Americana de Enfermagem, Ribeirão Preto, v. 8, n. 4, p. 7-14, ago. 2000.

OLIVEIRA, R. A.; CIAMPONE, M. H. T. Qualidade de vida de estudantes de enfermagem: a construção de um processo e intervenções. Revista da Escola de Enfermagem da USP, São Paulo, v. 42, n. 1, p. 57-65, mar. 2008.

PETTENGILL, M. A. M.; NUNES, C. B.; BARBOSA, M. A. M. Professor e aluno compartilhando da experiência de ensino-aprendizagem: a disciplina de enfermagem pediátrica da Universidade Federal de Mato Grosso do Sul. Revista Latino-Americana de Enfermagem, Ribeirão Preto, v. 11, n. 4, p. 453-460, jul./ago. 2003.

PIZÃO, M. F.; MARTINO, M. M. F. Estudo do sono em estudantes universitários de enfermagem. 2005. In: CONGRESSO INTERNO DE INICIAÇÃO CIENTÍFICA DA UNINCAMP, 13., set. 2005. Campinas. Anais... Campinas: UNICAMP, 2005.

RIBEIRO, M. I. L. C.; PEDRÃO, L. J. O ensino de enfermagem no Brasil: enfoque na formação de nível médio. Revista Nursing, v. 82, n. 8, p. 124-128, mar. 2005.

SANTOS, L. V. et al. Percepção de estudantes da área da saúde sobre abuso de álcool no meio acadêmico. In: ENCONTRO DE ESPECIALISTAS EM ENFERMAGEM PSIQUIÁTRICA, 5., 2000. Ribeirão Preto. Anais... Ribeirão Preto: FIERP/EERP - USP/FAPESP, 2000.

SAUPE, R. Qualidade de vida de estudantes de Enfermagem conforme escala de Flanagan. Ciência, Cuidado e Saúde, Maringá, v. 1, n. 2, p. 287-293, jul./ dez. 2002.

SAUPE, R. et al. Qualidade de vida dos acadêmicos de enfermagem. Revista Latino-Americana de Enfermagem, Ribeirão Preto, v. 12, n. 4, p. 636-642, jul./aug. 2003.

THE WHOQOL GROUP. The World Health Organization Quality of Life assessment (WHOQOL): position paper from the World Health Organization. Social Science and Medicine, Leicester, v. 41, n. 10, p. 1403-1409, 1995. 